\title{
Daptomycin: A Review 4 Years after First Approval
}

\author{
Robert Sauermann $^{a}$ Markus Rothenburger ${ }^{d}$ Wolfgang Graninger ${ }^{b}$ \\ Christian Joukhadarc \\ ${ }^{a}$ Department of Clinical Pharmacology, Division of Clinical Pharmacokinetics, ${ }^{b}$ Department of Internal Medicine I, \\ Division of Infections and Tropical Medicine, and ' Department of Internal Medicine II, Division of Pulmonology,

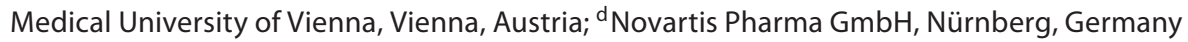

\author{
Key Words \\ Daptomycin • Clinical trial $\cdot$ Human $\cdot$ Efficacy $\cdot$ \\ Pharmacokinetics
}

\begin{abstract}
Daptomycin is the first approved member of a new class of antibiotics, namely the cyclic lipopeptides. Daptomycin has rapid bactericidal activity against Gram-positive pathogens. It acts by penetrating into the bacterial cell wall with consecutive formation of pores, loss of electrical membrane potential and inhibition of peptidoglycan synthesis. As the mode of action of daptomycin is 'concentration-dependent', the pharmacokinetic/pharmacodynamic indices that correlate best with its activity are the ratios of the peak concentration $\left(C_{\max }\right)$ to minimum inhibitory concentration $(\mathrm{MIC})$ or the area under the curve (24-hour AUC) to MIC. Daptomycin should be administered intravenously once daily, because adverse effects on skeletal muscle associated with an increase in plasma levels of creatine phosphokinase and myopathy were observed more frequently at shorter dosing intervals. Overall, the rate of adverse events during daptomycin therapy is comparable to that of other standard regimens. Daptomycin was shown to be not inferior to antimicrobial standard therapy and therefore was approved for complicated skin and skin structure infections at a dose of $4 \mathrm{mg} / \mathrm{kg}$, for Staphylococcus aureus bacteremia and right-sided endocarditis at a dose of $6 \mathrm{mg} / \mathrm{kg}$. Dosage regimens remain a mat-
\end{abstract}

\section{KARGER}

Fax +41613061234

E-Mail karger@karger.ch

www.karger.com (c) 2008 S. Karger AG, Basel

0031-7012/08/0812-0079\$24.50/0

Accessible online at:

www.karger.com/pha ter of discussion, and an increase in the currently approved doses from 4-6 to 6-8 $\mathrm{mg} / \mathrm{kg}$ per day for severe infections seems promising. Though not approved up to now, daptomycin appears to be a treatment alternative for Gram-positive bone and joint infections based on clinical observations. Large international studies showed high susceptibility of relevant Gram-positive pathogens to daptomycin, even in multidrug-resistant strains. Thus, treatment of infections caused by Gram-positive cocci resistant to other antimicrobial drugs is a potential indication of daptomycin. Since glycopeptides and daptomycin have the same target site, there appears to be a risk of reduced susceptibility to both drugs after consecutive use. Therefore, daptomycin should be used with caution for treatment of vancomycin-resistant isolates or after prior vancomycin (glycopeptide) therapy. This review describes the history, mechanism of action, susceptibility, recent discoveries and clinical experience regarding daptomycin, discussing its current role in the field of infectious diseases.

Copyright $\odot 2008$ S. Karger AG, Basel

\section{Introduction and History}

Daptomycin is the first approved member of an old class of antibiotics, the cyclic lipopeptides. It is a 13-amino acid compound which is derived from fermentation by a nonribosomal peptide synthetase mechanism of Strep- 


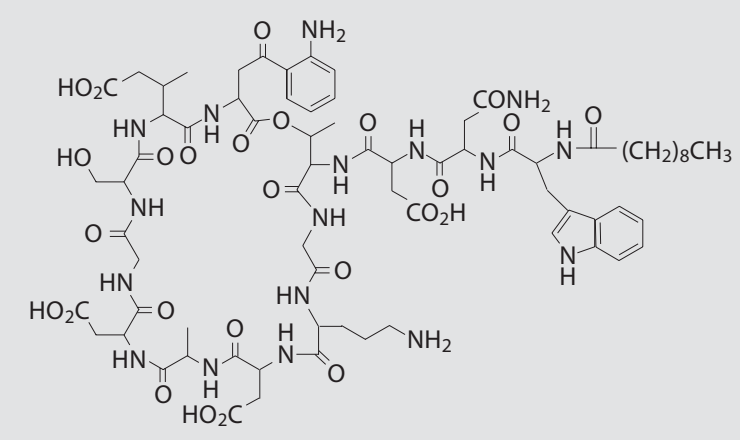

Fig. 1. Structure of daptomycin.

tomyces roseosporus [1]. The compound was discovered by Eli Lilly and Company in the 1980s and designated LY 146032, dapcin or cidecin in earlier years. Daptomycin showed promise in 19 phase 1 and two phase 2 clinical studies which were conducted in the 1980s and early 1990s involving more than 370 subjects [2]. Unexpected treatment failures which occurred in patients with bacteremia and endocarditis were attributed to inadequately low doses, suggesting higher dosing $[3,4]$. However, reversible adverse effects on skeletal muscle, including elevated creatine phosphokinase (CPK) levels, muscle weakness, and myalgia were reported after dosing every $12 \mathrm{~h}$. Overall, Eli Lilly was not satisfied with the clinical results observed with the twice-daily dosing regimens utilized in these studies and ceased further research activities. In 1997, worldwide development and commercialization rights to daptomycin were acquired by Cubist Pharmaceuticals. New attempts to investigate and develop daptomycin were favored by the dramatically increasing emergence of bacterial resistance among Gram-positive bacteria in the 1990s, like methicillin-resistant Staphylococcus aureus (MRSA). The pronounced trend towards higher resistance rates of clinically important bacterial species essentially contributed to reassessment of the benefit-risk ratios of new antimicrobial agents. Moreover, it was discovered that once-daily dosing of daptomycin in dogs, though leading to higher peak levels, causes significantly less frequent $\mathrm{CPK}$ increase and myopathy than thrice-daily dosing [5]. Thus, it turned out that skeletalmuscle effects are minimized if the total daily dose is administered as one single short infusion every $24 \mathrm{~h} \mathrm{[5].}$ Following successful phase 3 studies, daptomycin was approved for treatment of complicated skin and skin structure infections (SSSI) in the United States of America in
November 2003 at a dose of $4 \mathrm{mg} / \mathrm{kg}$ of body weight under the trade name Cubicin ${ }^{\circledR}$. Since 2006 daptomycin is marketed in several European Union member states by Novartis Pharmaceuticals following its acquisition of Chiron Pharmaceuticals, which previously held European licenses. Currently, daptomycin is going to be approved also for therapy of $S$. aureus blood stream infections at a dose of $6 \mathrm{mg} / \mathrm{kg}$.

\section{Chemistry and Mode of Action}

Daptomycin is a cyclic 13-residue lipopeptide, namely $\mathrm{N}$-decanoyl-L-tryptophyl-D-asparaginyl-L-aspartyl-Lthreonylglycyl-L-ornithyl-L-aspartyl-D-alanyl-L-aspartylglycyl-D-seryl-threo-3-methyl-L-glutamyl-3-anthraniloyl-L-alanine-lactone (fig. 1). It is provided for clinical purposes as lyophilized powder for the preparation of infusion solution [6].

The suggested mechanism of action consists of daptomycin insertion into the Gram-positive bacterial cytoplasmic membrane, whereas Gram-negative bacteria are not affected, probably due to their outer membrane [7]. A number of hydrophobic moieties are clustered at one end of the daptomycin molecule, while neutral polar and anionic residues are localized at the other end, leading to amphipathicity of the molecule. These features suggest a mode of action in which the large hydrophobic cluster of the lipopeptide interacts with the acyl chain region of the bacterial membrane. The interaction of daptomycin with the bacterial membrane is calcium-dependent. Calcium ions neutralize the anionic charges and favor association with the membrane head groups. Calcium binding increases the amphipathicity and the solvent-exposed hydrophobic surface [8]. Upon association with lipid membranes, daptomycin undergoes calcium-dependent conformational changes [8]. Once inserted into the membrane significant perturbations ensue, including lipid flip-flop [8]. The binding of calcium ions also causes the core decapeptide lactone to draw inwards. This not only facilitates further drug penetration into the membrane but also results in oligomerization of the daptomycin peptide. Oligomerization is followed by disruption of the functional integrity of the cytoplasmic membrane with formation of pores, triggering membrane leakage, release of intracellular ions and rapid cell death [7]. In addition, it was observed in Bacillus megaterium that active transport of cell wall amino acids, as well as formation of sugar-peptide precursors and peptidoglycans are inhibited by disruption of the transmembrane electrochemical 
gradient [9]. Jung et al. [8], in contrast, provided evidence that membrane depolarization occurs after cell death and is a consequence rather than a cause of the bactericidal activity of daptomycin. Howsoever, daptomycin has bactericidal activity, and bacterial killing by daptomycin is greater when the organism is exposed to the drug while in logarithmic growth than in stationary growth phase [4]. However, in contrast to other antimicrobial agents, the killing rates achieved with daptomycin during stationary growth are remarkably high [10].

Pharmacodynamic animal studies indicated that daptomycin has a concentration-dependent mode of action. This means that efficacy of daptomycin is best correlated with its peak concentration $\left(\mathrm{C}_{\max }\right)$ or 24 -hour area under the concentration versus time curve (AUC) in relation to the minimum inhibitory concentration (MIC) of the bacterial strain, rather than with the time the drug concentrations exceed MIC (time above MIC) [11-13]. In mice, the $\mathrm{C}_{\max } / \mathrm{MIC}$ ratios (using total drug concentrations) required for a bacteriostatic effect against $S$. aureus isolates ranged from 59 to 94, while the 24-hour AUC/MIC ratios to achieve bacteriostasis ranged from 388 to 537 . It is appropriate to apply the required magnitude of these indices obtained in mice to humans, because the extent of protein binding is identical in both species [12]. Thus, for humans the mean total $C_{\max }$ values of $99 \pm 12 \mu \mathrm{g} / \mathrm{ml}$ [14] and 24-hour AUC values of $598 \pm 110 \mu \mathrm{g} \times \mathrm{h} / \mathrm{ml}[15]$ measured at doses of $6 \mathrm{mg} / \mathrm{kg}$ can be related to the MICs inhibiting $90 \%$ of tested staphylococcal strains $\left(\mathrm{MIC}_{90}\right)$ of $0.5 \mu \mathrm{g} / \mathrm{ml}$ (table 1). According to these indices sufficient drug efficacy can be expected in the majority of patients. If the MICs of infecting strains exceed 0.5-1 $\mu \mathrm{g} /$ $\mathrm{ml}$, dose adjustment might be necessary to ensure that target AUCs are achieved.

Garrison et al. [3] evaluated the impact of protein binding on bacterial killing using an in vitro pharmacodynamic model. Serum concentration-versus-timecurves of patients receiving $6 \mathrm{mg} / \mathrm{kg}$ daptomycin as intravenous infusion were simulated in vitro in the presence and absence of $4 \%$ albumin. The average time required for a $99 \%$ kill of $S$. aureus was $0.3 \mathrm{~h}$ without albumin whereas it was $0.9 \mathrm{~h}$ in the presence of albumin. The fact that the presence of protein diminishes bactericidal activity is not surprising as daptomycin has a plasma protein binding of approximately $91.7 \%$ [16].

The postantibiotic effect (PAE) is a pharmacodynamic parameter which is defined as the length of time that bacterial growth is suppressed following brief exposure to an antibiotic. Daptomycin staphylococcal PAE ranged from approximately 1.1 to $6.2 \mathrm{~h}$, with a mean of $2.5 \mathrm{~h}$ [17,
Table 1. Susceptibility of relevant pathogens to daptomycin

\begin{tabular}{lll}
\hline Pathogen & MIC $_{90}, \mu \mathrm{g} / \mathrm{ml}$ & Reference \\
\hline MSSA & $0.25 / 0.5$ & {$[29] /[37]$} \\
MRSA & 0.5 & {$[29] /[37]$} \\
Coagulase-negative staphylococci & 0.5 & {$[37] /[36]$} \\
VS E. faecium & $2 / 4$ & {$[29] /[37]$} \\
VR E. faecium & $2 / 4$ & {$[29] /[37]$} \\
VS E. faecalis & 2 & {$[29] /[37]$} \\
VR E. faecalis & $4 / 2$ & {$[29] /[37]$} \\
Beta-hemolytic streptococci & 0.25 & {$[36]$} \\
Viridans streptococci & 0.5 & {$[36]$} \\
S. agalactiae & 0.25 & {$[37]$} \\
Corynebacterium spp. & 0.25 & {$[36]$} \\
Listeria spp. & 2 & {$[36]$} \\
\hline
\end{tabular}

VS = Vancomycin-susceptible; VR = vancomycin-resistant.

18]. The effect of subinhibitory drug levels following suprainhibitory levels which persist between dosing intervals is referred to as the postantibiotic sub-MIC effect (PA-SME). Staphylococcal PA-SME effects at 0.4 times the MIC ranged from $3.0 \mathrm{~h}$ to $>12 \mathrm{~h}$ [17]. PA-SMEs and PAEs for S. aureus and Enterococcus faecalis were dosedependent, and it was concluded that the relatively high PA-SME and PAE values found in vitro support oncedaily dosing of daptomycin $[17,18]$.

Compared to vancomycin or gentamicin, daptomycin was more effective in preventing bacterial adherence to the surfaces of medical devices, and biofilm formation $[19,20]$. Moreover, daptomycin was more active in inhibiting bacterial colonization of preexisting biofilms than other antimicrobial drugs [21]. Further in vitro experiments indicated that daptomycin is also active against $S$. aureus and Streptococcus epidermidis present in biofilms $[19,20]$. These properties suggest its utilization as catheter lock solution. Clinical studies, however, are required to test the in vivo efficacy in this indication.

Synergistic or additive effects against $S$. aureus were observed in vitro between daptomycin plus gentamicin, and additive effects were seen for daptomycin plus rifampicin [22]. Others, in contrast, found that the addition of gentamicin does not enhance the bactericidal activity of daptomycin against staphylococcal and enterococcal isolates [23]. According to in vitro experiments daptomycin plus either rifampicin, oxacillin, or fosfomycin had synergistic effects against the majority of enterococcal strains tested $[24,25]$. Surprisingly, daptomycin combined with either oxacillin, ampicillin-sulbactam, ticarcillin-clavu- 
lanate, or piperacillin-tazobactam also showed synergy against MRSA [26]. Different explanations for the synergistic effect of these beta-lactams against resistant bacteria have been provided. It was speculated that either daptomycin alters peptidoglycan precursors in a manner that penicillin binding protein $2^{\prime}$ might be unable to perform its cross-linking function in the presence of the beta-lactam, or that daptomycin affects the functionality of factors which are essential for methicillin resistance [26]. Alternatively, beta-lactams might enhance the activity of daptomycin. Though in vivo data are still required, these in vitro data indicate that the combination of daptomycin with beta-lactams could be reasonable also in S. aureus infections. Up to now, antagonism between daptomycin and other antimicrobial drugs has not been reported.

Exposure of murine macrophages to clinical S. aureus isolates in the presence of daptomycin led to a dampened inflammatory response with diminished secretion of tumor necrosis factor and reduced accumulation of inducible oxide synthase protein in comparison with addition of vancomycin or oxacillin [27]. Since excessive production of these inflammatory mediators can contribute to the development of sepsis in infection patients, the impact of this effect deserves further investigation.

\section{Antibacterial Spectrum and in vitro Susceptibility}

In general, daptomycin is active against a broad spectrum of Gram-positive bacteria, while there is no activity against Gram-negative pathogens. Activity against distinct anaerobic species was observed. To date, the clinically most relevant pathogens are coagulase-positive and coagulase-negative staphylococci, as well as streptococci and enterococci. Importantly, daptomycin was observed to be active also against a number of pathogens that are resistant to other antibiotics $[4,28]$.

When performing broth microdilution susceptibility tests calcium must be supplemented in order to obtain correct results. The activity of daptomycin is enhanced two- to fourfold by increasing the calcium concentration in Mueller-Hinton broth from the usual in vitro concentration of 20-25 to $50 \mathrm{mg} / \mathrm{l}$, a level which closely approximates ionized calcium levels in human serum $[29,30]$. For most pathogens the breakpoint of susceptibility is defined by an MIC of $\leq 1 \mu \mathrm{g} / \mathrm{ml}$, whereas $E$. faecalis isolates are considered susceptible if the MIC is $\leq 4 \mu \mathrm{g} / \mathrm{ml}$ [31].

In spite of calcium substitution, testing of $S$. aureus strains by disk diffusion failed to detect strains catego- rized as nonsusceptible by the microdilution method [32-34]. Thus, disk diffusion tests do not provide reproducible results and are therefore currently not recommended for susceptibility testing of daptomycin. Consequently daptomycin disks were removed from the market by Cubist Pharmaceuticals and the respective disk diffusion breakpoints were removed from the CLSI M100 standard in 2006 [35].

In contrast, the epsilon test (Etest) method using daptomycin strips with calcium supplementation $(\mathrm{AB}$ Biodisk, Solna, Sweden) provided a relatively good correlation with results of broth microdilution (95\% of values within $\pm 1 \log _{2}$ dilution) [34]. However, among selected strains identified as nonsusceptible by broth microdilution, $9.8 \%$ of tested isolates were classified as susceptible by Etest. This discrepancy was in part attributed to the lack of an intermediate range for classifying daptomycin susceptibility [34]. Nevertheless, the Etest was judged to be a reliable method by different study authors [32, 34]. Regardless of the method used for susceptibility testing, we recommend careful clinical and microbiologic monitoring if the MIC values of treated strains are close to the breakpoint.

Large multicenter studies investigating the clinically most relevant pathogens in Europe, North and South America showed that all staphylococcal and $>95 \%$ of enterococcal isolates, including glycopeptide-susceptible and -resistant enterococci as well as MRSA were susceptible to daptomycin in vitro (table 1) [29, 36, 37]. The MICs inhibiting $90 \%$ of tested strains $\left(\mathrm{MIC}_{90}\right)$ were $0.25-$ $0.5 \mu \mathrm{g} / \mathrm{ml}$ for staphylococcal and streptococcal isolates, and $2-4 \mu \mathrm{g} / \mathrm{ml}$ for enterococcal isolates [29, 36, 37]. Multidrug-resistant isolates were defined as concurrent resistance to three or more agents from different classes. In multidrug-resistant and non-multidrug-resistant isolates of $S$. aureus, enterococci, and S. pneumoniae, there was no major difference in the MIC distributions of daptomycin, i.e. modal MICs $\leq 1$ doubling dilution.

Importantly, isolates of Streptococcus pyogenes, Streptococcus agalactiae, streptococci of groups $\mathrm{C}$ and $\mathrm{G}$, viridans streptococci and Listeria monocytogenes were susceptible to daptomycin in vitro, independent of vancomycin or methicillin resistance [38]. Susceptibility of less frequent Gram-positive pathogens Erysipelothrix rhusiopathiae, Corynebacterium spp., Abiotrophia/Granulicatella sp., Rothia mucilaginosus and Gemella morbillorum was reported, with MIC values ranging from $\leq 0.125$ to $2 \mu \mathrm{g} / \mathrm{ml}$ [39]. Daptomycin was active in vitro also against some Gram-positive anaerobes including Clostridium perfringens, Clostridium difficile, Peptostreptococcus spp., 
Eubacterium spp., Propionibacterium spp., Leuconostoc spp., Finegoldia magna and Pediococcus spp. [28, 40-42]. In contrast, daptomycin had decreased activity against Actinomyces, Clostridium ramosum, Eubacterium lentum and Lactobacillus plantarum strains [42].

\section{Pharmacokinetics}

Daptomycin pharmacokinetics (PK) follows a twocompartment model in plasma with first-order elimination $[14,43,44]$. In healthy volunteers daptomycin PK is linear and dose-proportional at doses up to $12 \mathrm{mg} / \mathrm{kg}$ [45]. The distribution phase is not complete until $4-6 \mathrm{~h}$ after administration. At steady state, after 7 daily doses of $6 \mathrm{mg} / \mathrm{kg}$, mean plasma elimination half-life was $8.9 \pm$ $1.3 \mathrm{~h}$ in healthy volunteers [14]. The volume of distribution was $0.104 \pm 0.013$ liters $/ \mathrm{kg}$, corresponding with a predominantly extracellular distribution and high protein binding. The concentrations of ${ }^{14} \mathrm{C}$-labeled daptomycin were much higher in plasma than in whole blood, indicating that little of the drug crosses into erythrocytes [15]. In plasma the mean AUC from zero to infinity $\left(\mathrm{AUC}_{0-\text { inf }}\right)$ was $747 \pm 91 \mu \mathrm{g} \times \mathrm{h} / \mathrm{ml}, \mathrm{C}_{\max }$ was $98.6 \pm$ $12.0 \mu \mathrm{g} / \mathrm{ml}$ at steady state (doses of $6 \mathrm{mg} / \mathrm{kg}$ ). Plasma protein binding is reversible and approximates 91.7\% [16]. Protein binding was not concentration-dependent at daptomycin concentrations between 2.5 and $80 \mu \mathrm{g} / \mathrm{ml}$ [16]. Daptomycin is mainly excreted by the kidneys, with a plasma clearance (CL) of about $7-9 \mathrm{ml} / \mathrm{h} / \mathrm{kg}$ and a renal CL of $4-7 \mathrm{ml} / \mathrm{h} / \mathrm{kg}$. Importantly, metabolization by liver microsomes is limited, with only minimal involvement of the cytochrome P-450 isoenzymes [15].

In order to identify factors contributing to interindividual variability of daptomycin $\mathrm{PK}$, a population $\mathrm{PK}$ analysis was performed with data from 15 clinical trials considering over 30 covariates [46]. Daptomycin plasma $\mathrm{CL}$ was a function of renal function, body temperature and sex. Of these, renal function contributed most significantly to interindividual variability. While drug CL varied linearly with the estimated creatinine CL, the relationship to sex and temperature was of doubtful impact. Concluding, this analysis supported dosing on a milligram per kilogram body weight basis with suggested modified regimens only for patients with severe renal disease.

Impact of Age, Obesity and Drug Abuse

A single-dose study compared the PK of daptomycin in young ( $18-30$ years) versus old ( $\geq 75$ years) healthy vol- unteers, detecting higher values of $\mathrm{AUC}_{0 \text {-inf }}$ and terminal half-life in the older group [43]. This was interpreted as a result of changes in renal function, i.e. impaired renal CL in subjects $\geq 75$ years of age. No differences were observed for $C_{\max }$ and the volume of distribution. It was concluded that no dose adjustment is necessary only due to age. A study in children and adolescents $\leq 18$ years is currently being performed in the United States of America.

A more recent study showed that in moderately obese and morbidly obese subjects, absolute volumes of distribution and plasma CL of daptomycin were higher compared to matched nonobese controls [47]. Drug exposure was increased in obese subjects, as expressed by an approximately $25 \%$ increase in $\mathrm{C}_{\max }$ and an approximately $30 \%$ increase of AUC in plasma compared to nonobese controls. Thus, dosing based on body weight results in higher $\mathrm{C}_{\max }$ and AUC values in obese subjects [48]. However, this increase was well within the range that was previously determined to be safe and well tolerated, and study authors concluded that no dose adjustment is required for morbidly obese patients [47].

In intravenous drug abusers peak serum concentrations were observed to be lower than in healthy volunteers [4]. It was speculated that this finding may be attributed to the descriptively higher renal drug CL in drug abusers. Alternatively the descriptively larger volume of distribution found in drug abusers was reported as a possible cause of lower drug serum concentrations. The reasons for the larger volume of distribution and higher renal CL in intravenous drug abusers, however, were unclear.

\section{Penetration into Deep Compartments}

The penetration of daptomycin into cantharidin-induced inflammatory blister fluid in healthy volunteers was investigated by Wise et al. [49]. The mean peak concentrations were $77.5 \mu \mathrm{g} / \mathrm{ml}$ in plasma versus $27.6 \mu \mathrm{g} / \mathrm{ml}$ in inflammatory fluid, with terminal elimination halflives of 7.74 and $13.2 \mathrm{~h}$, respectively. The mean ratio of 24-hour AUC in inflammatory fluid to that in plasma was 0.68 , suggesting good penetration into inflammatory bodily fluids. The relatively high drug concentrations in blister fluid in spite of high plasma protein binding (approximately $90 \%$ ) could be explained by the considerable amount of protein in blister fluid, where also remarkable drug amounts are bound.

Daptomycin passes the blood-brain barrier only to a small degree. In experimental meningitis in rabbits, penetration of daptomycin was between 4.4 and $7.5 \%$. Pene- 
tration into inflamed meninges was higher than into noninflamed meninges (2\%) within the first $8 \mathrm{~h}$ [50]. According to the authors daptomycin at a dose of $15 \mathrm{mg} / \mathrm{kg}$ was clinically and microbiologically more effective in experimental meningitis than a standard regimen with ceftriaxone plus vancomycin [50,51]. Daptomycin doses used in the meningitis model, however, were quite high, and it is too early at this stage to apply the rabbit data to clinical practice.

\section{Dosing Regimens}

Dosing of daptomycin is based on individual body weight. Daptomycin is currently approved at a dose of 6 $\mathrm{mg} / \mathrm{kg}$ of body weight for therapy of $S$. aureus bacteremia, including right-sided endocarditis, and a dose of $4 \mathrm{mg} / \mathrm{kg}$ for complicated skin and skin structure infections. Since daptomycin has a concentration-dependent mode of action (i.e. its efficacy depends on $\mathrm{C}_{\max }$ or AUC), and in order to reduce adverse side effects the entire daily dose should be administered as one single intravenous short infusion. In addition, once-daily administration might favor patient compliance, compared to agents requiring multiple daily doses. It was reported that daptomycin was safe also if administered as a bolus of $6 \mathrm{mg} / \mathrm{kg}$ over $2 \mathrm{~min}$ [52], which also might promote its use for outpatient parenteral antibiotic therapy of community-acquired infections. Adverse events possibly related to bolus injection were dizziness and headache.

In patients with severe or terminal renal insufficiency (creatinine $\mathrm{CL}<30 \mathrm{ml} / \mathrm{min}$ ) and in subjects undergoing hemodialysis or peritoneal dialysis daptomycin should be used cautiously because elimination half-life and AUC are increased by two- to threefold. If creatinine $\mathrm{CL}$ is $<30$ $\mathrm{ml} / \mathrm{min}$ dosing intervals should be extended to $48 \mathrm{~h}$, with administration after dialysis if possible. For patients receiving continuous renal replacement therapy the daptomycin dose should be the same as recommended for patients with a creatinine $\mathrm{CL}<30 \mathrm{ml} / \mathrm{min}$ [53].

A matched controlled study in patients with moderate liver impairment showed similar PK parameters as in the healthy group after a single dose of $6 \mathrm{mg} / \mathrm{kg}$ total body weight [44]. Since metabolization by liver microsomes is marginal, patients with moderate hepatic impairment (Child-Pugh class B) do not require adjustment of daptomycin dose regimen.

Clinical experience with 1 patient and a study involving 12 healthy volunteers indicate that doses of $12 \mathrm{mg} / \mathrm{kg}$ can be administered over $\geq 14$ days without any toxicity
$[45,54]$. Although currently this dosing regimen cannot be recommended, these experiences illustrate that higher doses are indicated in select cases and that such doses might be approved in the future (e.g. 6-8 $\mathrm{mg} / \mathrm{kg}$ for deepseated staphylococcal infections). Moreover, higher doses are expected to impede emergence of bacterial resistance.

\section{Efficacy in Different Indications}

\section{Severe Skin Infections}

The efficacy of daptomycin for treatment of SSSI was investigated in large international clinical trials [55]. A total number of 1,092 patients with SSSI including wound infections, abscesses, diabetic and other ulcera were randomized to receive either daptomycin at a dose of $4 \mathrm{mg} /$ $\mathrm{kg}$ once daily or standard therapy. Standard therapy consisted of either cloxacillin, oxacillin, nafcillin, flucloxacillin, or, if risk of MRSA was suspected, vancomycin. Success of treatment was defined as clinical cure, disappearance of infection signs and symptoms or improvement. Nine hundred and two patients were evaluable and therapeutic equivalence was found with an overall success rate of $83.4 \%$ for daptomycin, and of $84.2 \%$ for the control group. Both groups were equivalent with regard to the distribution of the pathogens methicillin-susceptible S. aureus (MSSA), MRSA, S. pyogenes, S. agalactiae, and Streptococcus dysgalactiae. Though not being a main outcome variable, clinical success occurred more rapidly with daptomycin than with comparable drugs, with success rates on day 7 of 63 vs. 33\%, respectively. A prospective study with randomization to either daptomycin or comparable antibiotic (vancomycin or semisynthetic penicillin) for SSSI by Lipsky and Stoutenburgh [56] showed essentially the same result, i.e. clinical and microbiological success rates were equivalent.

\section{Bacteremia and Endocarditis}

The effect of daptomycin (6 mg/kg of body weight) in the therapy of bacteremia and endocarditis caused by $S$. aureus was investigated in a randomized clinical trial in comparison with standard therapy [57]. In the control group an antistaphylococcal penicillin plus gentamicin were used for the treatment of MSSA, while vancomycin was utilized against MRSA. This trial in 246 patients demonstrated noninferiority of daptomycin for treatment of $S$. aureus right-sided endocarditis and $S$. aureus bacteremia in both the intention-to-treat population and in the patient group with documented adherence to the
84

Pharmacology 2008;81:79-91
Sauermann/Rothenburger/Graninger/ Joukhadar 
protocol. For the intention-to-treat population, overall success rates were $44.2 \%$ in the daptomycin group and $41.7 \%$ in the control group. Subgroup analysis showed a descriptive advantage of daptomycin for MRSA, and a descriptive advantage of standard therapy for MSSA. Daptomycin had a nonsignificantly higher rate of microbiologic failure (15.8\%), with isolation of S. aureus strains becoming less susceptible to daptomycin during treatment in 7 of 19 patients. Low success rates in both groups were observed for left-sided endocarditis (1 of 9 patients in the daptomycin group, 2 of 9 patients in the standard therapy group). The overall rate of adverse events was lower in the daptomycin group without reaching statistical significance. Renal dysfunction occurred in $11.0 \%$ of patients who received daptomycin and in $26.3 \%$ of patients who received standard therapy $(p=0.004)$, a finding essentially attributed to the thrice-daily administration of gentamicin to patients who were randomized to the control group. Limitations of the clinical trial were the short follow-up period of 42 days after therapy completion, and the relatively small number of cases of endocarditis (64 cases of endocarditis among 246 patients, of these 18 cases of left-sided endocarditis).

A retrospective review was performed of 31 patients with Gram-positive bacteremia or infective endocarditis who received daptomycin therapy at doses of $4-6 \mathrm{mg} / \mathrm{kg}$ intravenously based on the practitioner's discretion [58]. Eleven patients had MRSA, 11 patients had vancomycinresistant enterococci, and 7 patients had MSSA infection. Clinical resolution was achieved in 24 patients (77\%), including all MRSA patients. Seven patients died, six of whom had vancomycin-resistant enterococci.

The Cubicin Outcomes Registry and Experience (CORE) is a multicenter retrospective observational chart review of daptomycin patient outcomes. The CORE group reported 61 patients with non-catheter-related bacteremia who were treated with daptomycin [59]. Of these, $46 \%$ received concurrent antibiotic therapy, mostly beta-lactams. Overall, clinical success defined as improvement or cure was achieved in $79 \%$.

There is limited data about the application of daptomycin in neutropenic bacteremia with vancomycin-resistant Enterococcus faecium, where cure was achieved with daptomycin in 4 of 9 patients [60]. A case of pacemakerinduced $S$. aureus mitral valve acute bacterial endocarditis with persistent bacteremia from a coronary stent was cured with prolonged high-dose daptomycin therapy 12 $\mathrm{mg} / \mathrm{kg}$ for 41 days without occurrence of any toxicity [54]. Successful 6-week treatment of Corynebacterium striatum endocarditis with daptomycin plus rifampicin was reported in a woman with hemodialysis-dependent chronic renal failure [61].

\section{Bone and Joint Infections}

CORE retrospectively analyzed 67 patients with osteomyelitis treated with daptomycin after therapy [62]. Median therapy duration was 35 days (range 1-546 days) with a mean initial dose of $5.3 \mathrm{mg} / \mathrm{kg}$. Out of 67 patients 42 (63\%) were cured, 13 patients improved. Failure was observed in 7 patients, 5 were nonevaluable. There was a trend towards higher failure rates (27\%) for doses $\leq 4 \mathrm{mg} /$ $\mathrm{kg}$, compared to doses $>4 \mathrm{mg} / \mathrm{kg}$ with a failure rate of $\leq 7 \%$. It was concluded that daptomycin may be useful for osteomyelitis, but that further prospective trials are necessary to properly answer this question.

Moreover, CORE retrospectively analyzed 26 of 31 patients after treatment for septic arthritis [63]. The mean daptomycin treatment duration was 29 days. Eighty-eight percent had received another antibiotic prior to daptomycin. The change to daptomycin was performed in most cases due to allergy or intolerance of vancomycin. Fifty percent received concomitant antibiotics in addition to daptomycin. Cure was observed in $58 \%$, improvement in $38 \%$ of patients. No failure occurred in patients with Gram-positive pathogens, the only failure was observed in a case where the infection turned out to be caused by Gram-negative pathogens in the culture. Another retrospective analysis of 9 patients diagnosed with Gram-positive bone and joint infection showed that 8 were successfully treated [64], indicating that joint infections may become an indication for daptomycin therapy.

Twelve patients with Gram-positive prosthetic joint infection, who could not receive vancomycin, were prospectively monitored [65]. All completed a 6-week course of daptomycin. At follow-up after 7-13 months 6 patients had no clinical, laboratory or radiographic signs of recurrence. In 1 patient the first course failed and hardware removal was performed. Five patients had failure due to MRSA confirmed by culture. The authors concluded that effectiveness of daptomycin in prosthetic joint infection is uncertain, especially when hardware is retained.

\section{Mixed Indications}

Finally, CORE retrospectively reviewed outcomes of 810 patients with differently located infections receiving daptomycin [66]. Of these, $56 \%$ had SSSI, $18 \%$ had bacteremia, $13 \%$ had osteomyelitis. Seventy-six percent had received other antibiotics prior to daptomycin. Daptomycin doses were $4 \mathrm{mg} / \mathrm{kg}$ in $51 \%$ and $\geq 6 \mathrm{mg} / \mathrm{kg}$ in $37 \%$ of 
patients. Overall, $92 \%$ of patients achieved clinical success, defined as improvement or cure. Highest success rates were detected in SSSI (95\%) and osteomyelitis (93\%). Lower success rates were described for foreign body infections (88\%) and endocarditis (76\%). In the data set that was analyzed success rates were independent of the applied dose. In patients with impaired renal function (creatinine $\mathrm{CL}<30 \mathrm{ml} / \mathrm{min}$ ) success rates were lower $(82 \%)$ than in patients with a creatinine $\mathrm{CL} \geq 30 \mathrm{ml} / \mathrm{min}$ (94\%). However, reduced renal function might only reflect the higher risk and morbidity of these patients.

\section{Pneumonia}

Phase III clinical trials were conducted for the treatment of hospitalized patients with community-acquired pneumonia. Despite potent bactericidal activity against S. pneumoniae in vitro, daptomycin failed to achieve superiority over ceftriaxone ( 79 vs. $87 \%$ efficacy) in humans [67]. Failure to treat bronchial-alveolar pneumonia by $S$. pneumoniae was observed in a mouse model of infection [67], whereas it was assessed to be as efficient as vancomycin in a hamster pneumonia model using $S$. aureus [68]. In vitro experiments showed that daptomycin interacts with pulmonary surfactant, resulting in inhibition of antibacterial activity. This interaction is specific to daptomycin and consistent with its mechanism of action. Daptomycin irreversibly inserts into the surfactant aggregates, resulting in sequestration of the antibiotic, rendering it inactive [67]. These experiments do not answer some crucial questions such as, how is daptomycin eliminated from the lung when bound to surfactant? Is its accumulation in surfactant saturable? Thus, daptomycin is currently not recommended for monotherapy of pneumonia and subsequent studies are necessary to clarify these open questions. However, the situation appears to be different for abscesses in the lung caused by $S$. aureus where surfactant is not expected to interact with the drug to a relevant degree.

Summarizing this entire section, therapeutic equivalence of daptomycin in comparison with standard antibiotics was proven for Gram-positive SSSI and S. aureus bacteremia and right-sided endocarditis. Though definitive evidence from randomized trials is missing, there is data to support daptomycin as a useful treatment option for Gram-positive prosthetic valve and pacemaker infections, as well as for osteomyelitis and joint infections. Data on urinary tract and foreign body infections is scarce, but daptomycin might be eligible in some cases. From the available data, it is tempting to speculate that daptomycin may also be adequate for treatment of intra- abdominal abscesses or diabetic foot infections in combination with other antimicrobial agents. This, however, needs to be confirmed by clinical studies.

\section{Emergence of Daptomycin Resistance}

A study was performed in vitro to determine the emergence of bacterial resistance using serial passages in daptomycin-containing broth and chemical mutagenesis [69]. The selected mutants had MICs which were 8- to 32fold higher than for the parental strain. These MIC increases were considered relatively modest, comparable to previous findings for other antimicrobial drugs. MIC increases were stable during repeated passages in the absence of drug. There were mutants with normal growth rates, with reduced growth rates and with severe growth defects. Daptomycin remained bactericidal against all mutants at 8 times the MIC. These mutants did not demonstrate cross-resistance to vancomycin or ampicillin. Some mutants with increased MICs partly lost virulence in vivo $[69,70]$. In an animal model, emerging resistance was created by administering suboptimal doses $(1.5 \mathrm{mg} /$ $\mathrm{kg}$ ), whereas continued bactericidal activity persisted at doses of 6 and $10 \mathrm{mg} / \mathrm{kg}$, confirming the importance of adequately high dosing regimens [71].

Lately, clinical cases of emerging nonsusceptibility during daptomycin therapy were observed for E. faecium [72], methicillin-resistant S. epidermidis, methicillin-resistant Streptococcus sanguis (our own clinical observations), and MRSA [73-75], especially after prolonged drug exposure. Based on the increase of MICs during therapy observed in some cases, it was concluded that daptomycin susceptibility should be monitored during therapy [76], and that dosing should be adequately high. In spite of modestly rising MICs patients were treated successfully with daptomycin, if the medical management was adequate [77]. On the other hand, 1 case of decreased bacterial killing by daptomycin was described for a breakthrough isolate in spite of unchanged MICs [78].

Special attention was raised by the clinical observation of reduced staphylococcal susceptibility to daptomycin after prior vancomycin treatment, e.g., in a case with prosthetic joint infection and bacteremia caused by MRSA [79]. In this patient $S$. aureus persisted in repeated blood cultures during prolonged courses of vancomycin despite susceptibility to vancomycin as well as to daptomycin. However, after prolonged consecutive daptomycin therapy, susceptibility to both vancomycin and daptomycin decreased. Clinical cases of decreasing suscepti-
86

Pharmacology 2008;81:79-91
Sauermann/Rothenburger/Graninger/ Joukhadar 
bility of $S$. aureus to vancomycin by antibiotic pressure of daptomycin treatment or vice versa, associated with clinical treatment failure, were reported by various groups for differently located infection sites [33, 80-83]. Similar clinical observations were reported for enterococcal infections $[72,84]$. These findings were explained by the shared target of vancomycin and daptomycin. Although daptomycin and vancomycin have different mechanisms of action, both agents act on the bacterial cell wall. Staphylococcal cell wall thickening induced by prolonged exposure to vancomycin acts as a physical barrier to both daptomycin and vancomycin penetration of the cell, increasing the MICs of both antimicrobials [80]. The relative molecular weights of daptomycin $(1,620.67)$ and of vancomycin $(1,485.7)$ are comparatively high. A direct correlation between reduced daptomycin susceptibility, vancomycin resistance and cell wall thickening could be established in $53 \mathrm{~S}$. aureus strains. This observation was confirmed by genetic investigations proving that the levels of proteins which form part of the bacterial response to inhibition of peptidoglycan biosynthesis change similarly after exposure to either vancomycin or daptomycin [85]. Typical changes in the peptidoglycan composition occur in strains developing resistance to either vancomycin or daptomycin, i.e. a reduction in O-acetylation, which could lead to hydrophobicity and altered substrate properties towards cell wall-lytic enzymes [86].

In MRSA daptomycin has the same clinical success rates as in MSSA [57]. Thus, with the exception of vancomycin, reduced susceptibility to daptomycin by prior administration of other antimicrobial drugs has not been reported. Consequently, daptomycin is not the first therapeutic choice for treatment of $S$. aureus infections if reduced susceptibility to vancomycin (or teicoplanin) is suspected or documented [87]. Clinicians also should be aware of the fact that daptomycin has to be used with caution after failure of prolonged vancomycin therapy, even in vancomycin-susceptible pathogens.

\section{Safety}

The most frequent adverse events according to the manufacturer are anemia, constipation, diarrhea, nausea, vomiting, local injection site reactions, and headache. Less frequent, but important, are adverse effects on skeletal muscle which may appear as increases in CPK (MM isoenzymes), muscle pain, muscle weakness, or myositis during therapy, up to rhabdomyolysis. Among 534 patients with complicated SSSI receiving $4 \mathrm{mg} / \mathrm{kg}$ of dap- tomycin once daily $0.2 \%$ of the study population reported myopathy [55], which resolved after drug discontinuation $[55,88]$. Muscle pain may also occur without pronounced CPK elevation, apparently resolving after discontinuation [89]. Rates of drug-related CPK increase were $2.1 \%$ ( $\mathrm{n}=11$ of 534 patients) in the daptomycin group and $1.4 \%$ ( $\mathrm{n}=8$ of 558 patients) in the control group receiving standard therapy [55]. In healthy volunteers with once-daily dosing, only slight CPK increases with normalization of $\mathrm{CPK}$ values during ongoing therapy were observed [14]. Severe myopathy after once-daily daptomycin administration was described in 1 case, where daptomycin was started after discontinuation of simvastatin [90] and in another case during combined administration with voriconazole [91]. The mechanisms of skeletal muscle toxicity of daptomycin in humans are not known up to now. In dogs CPK increase correlated with alterations in muscle fibers, with a maximum on day 8 [5]. The heart muscle was histologically not affected in dogs [5]. A toxicodynamic study revealed that probability of CPK increase after 14 days of treatment is more likely (0.51) if trough levels are $\geq 25.7 \mathrm{mg} / \mathrm{l}$, while being much lower (0.06) if trough levels are $<25.7 \mathrm{mg} / 1$ [92]. Measuring of daptomycin trough levels, however, is not a routine procedure. The overall incidence of severe myopathy might seem acceptable, for instance in comparison with that of HMG-CoA reductase inhibitors, where its frequency is estimated to be $0.1 \%$ [93]. Nevertheless, before start and during daptomycin therapy weekly monitoring of CPK levels is recommended, with more frequent testing in cases of unexplained CPK elevations. According to the manufacturer daptomycin should be discontinued in patients with unexplained signs of myopathy in conjunction with CPK elevation $>1,000 \mathrm{U} / \mathrm{l}$, or in patients without marked symptoms who have marked elevations in CPK ( $\geq 5$ - to 10 -fold upper normal limit). Concomitant therapy with drugs that also may cause myopathy, rhabdomyolysis or CPK elevation should be avoided. For instance, consideration should be given to temporarily suspending the use of HMG-CoA reductase inhibitors, or cyclosporin during daptomycin therapy. The clinical combination of daptomycin plus fluoroquinolones, which also have a potential of causing tendopathy and myopathy, has not been investigated yet. Therefore, we suggest special precaution in this regard.

Peripheral neuropathy and renal insufficiency during daptomycin therapy were reported, however, the link with daptomycin is not clear. Possible hepatotoxicity, ending after discontinuation of daptomycin, was observed in 1 case [88]. If Gram-negative infections occur 
during daptomycin therapy they are obviously not covered by daptomycin therapy. A case of daptomycin-induced eosinophilic pneumonia was reported [94]. There are no data available concerning daptomycin in pregnancy. However, in animal experiments there was no evidence of harmful effects on pregnancy, prenatal development, birth, and postnatal development.

Overall, in the two large clinical trials the rate of side effects was equivalent to that of standard therapy $[55,57]$, with a nonsignificantly higher rate of adverse effects in the standard therapy group in one of these studies [57], essentially due to renal disorders caused by the addition of gentamicin. High-dose daptomycin administration of $12 \mathrm{mg} / \mathrm{kg}$ per day for 41 days to 1 patient and to 12 healthy volunteers over 14 days was not associated with any toxicity $[45,54]$.

\section{Summary}

Daptomycin belongs to a new class of cyclic lipopeptide antibiotics with rapid bactericidal activity against Gram-positive pathogens. Due to drug penetration into the bacterial cell wall consecutive formation of pores occurs with impairment of peptidoglycan synthesis and loss of the electrical membrane potential.

Daptomycin should be administered only once daily, because severe adverse effects were observed more frequently if infusions were administered every 8 or $12 \mathrm{~h}$. A once-daily daptomycin administration regimen is also supported by its 'concentration-dependent' activity. Accordingly, the pharmacokinetic/pharmacodynamic indices that correlate best with its activity are the ratios of $\mathrm{C}_{\max }$ to MIC and 24-hour AUC to MIC. Administration once daily suggests the use of daptomycin for outpatient fections. Dosing is performed according to body weight proved doses from $4-6$ to $6-8 \mathrm{mg} / \mathrm{kg}$ per day appears and for $S$. aureus bacteremia and right-sided endocarditis also for bone and joint infections caused by Gram-posidue to drug inactivation by surfactant. Treatment of in-

fections caused by Gram-positive strains resistant to other antimicrobial drugs may be an important indication of daptomycin. Though not approved, daptomycin might be considered also for pacemaker prosthetic valve or joint infections, osteomyelitis, or in patients requiring prolonged antibiotic therapy who have received linezolid for 28 days, as linezolid therapy is approved for a maximum period of 28 days only. Daptomycin should be used with caution for treatment of vancomycin-resistant isolates or after prior vancomycin therapy because glycopeptide antibiotics and daptomycin have the same target site, bearing the risk of reduced susceptibility to both drugs after consecutive use. If daptomycin is applied for mixed infections involving Gram-negative pathogens (e.g. diabetic foot) it has to be combined with an appropriate antimicrobial drug.

The rate of adverse effects is comparable to other standard antibiotics. The most important adverse effect during daptomycin therapy is increase in CPK levels in plasma and myopathy [5]. Therefore, at least weekly monitoring of CPK levels is recommended during therapy. Concomitant therapy with drugs that are associated with myopathy, rhabdomyolysis or CPK elevations should be avoided.

Large international studies in general showed high susceptibility of relevant Gram-positive pathogens to daptomycin, even in multidrug-resistant strains. Promising clinical results suggest that the use of daptomycin may be expanded to additional indications. In an era of emerging bacterial resistance to established antibiotics, daptomycin turns out to be a valuable treatment option for infections caused by Gram-positive pathogens. parenteral antibiotic therapy of community-acquired in$(4-6 \mathrm{mg} / \mathrm{kg})$ and has to be adjusted in patients with impaired renal function. Dosage of daptomycin is still a matter of discussion, and an increase in the currently apreasonable for severe infections due to recent clinical data.

Daptomycin is not inferior to standard therapy and is approved for the treatment of SSSI at a dose of $4 \mathrm{mg} / \mathrm{kg}$ at a dose of $6 \mathrm{mg} / \mathrm{kg}$. It seems to be a treatment alternative tive cocci, whereas it is not recommended for pneumonia

References
Sauermann/Rothenburger/Graninger/ Joukhadar 
-4 Rybak MJ, Bailey EM, Lamp KC, Kaatz GW: Pharmacokinetics and bactericidal rates of daptomycin and vancomycin in intravenous drug abusers being treated for gram-positive endocarditis and bacteremia. Antimicrob Agents Chemother 1992;36:1109-1114.

5 Oleson FB Jr, Berman CL, Kirkpatrick JB, Regan KS, Lai JJ, Tally FP: Once-daily dosing in dogs optimizes daptomycin safety. Antimicrob Agents Chemother 2000;44:29482953.

-6 Rotondi KS, Gierasch LM: A well-defined amphipathic conformation for the calciumfree cyclic lipopeptide antibiotic, daptomycin, in aqueous solution. Biopolymers 2005; 80:374-385.

-7 Silverman JA, Perlmutter NG, Shapiro HM: Correlation of daptomycin bactericidal activity and membrane depolarization in Staphylococcus aureus. Antimicrob Agents Chemother 2003;47:2538-2544.

-8 Jung D, Rozek A, Okon M, Hancock RE: Structural transitions as determinants of the action of the calcium-dependent antibiotic daptomycin. Chem Biol 2004;11:949-957.

$\checkmark 9$ Allen NE, Alborn WE Jr, Hobbs JN Jr: Inhibition of membrane potential-dependent amino acid transport by daptomycin. Antimicrob Agents Chemother 1991;35:26392642.

10 Lamp KC, Rybak MJ, Bailey EM, Kaatz GW: In vitro pharmacodynamic effects of concentration, $\mathrm{pH}$, and growth phase on serum bactericidal activities of daptomycin and vancomycin. Antimicrob Agents Chemother 1992;36:2709-2714.

-11 Dandekar PK, Tessier PR, Williams P, Zhang C, Nightingale CH, Nicolau DP: Determination of the pharmacodynamic profile of daptomycin against Streptococcus pneumoniae isolates with varying susceptibility to penicillin in a murine thigh infection model. Chemotherapy 2004;50:11-16.

-12 Safdar N, Andes D, Craig WA: In vivo pharmacodynamic activity of daptomycin. Antimicrob Agents Chemother 2004;48:63-68.

-13 Louie A, Kaw P, Liu W, Jumbe N, Miller MH, Drusano GL: Pharmacodynamics of daptomycin in a murine thigh model of Staphylococcus aureus infection. Antimicrob Agents Chemother 2001;45:845-851.

-14 Dvorchik BH, Brazier D, DeBruin MF, Arbeit RD: Daptomycin pharmacokinetics and safety following administration of escalating doses once daily to healthy subjects. Antimicrob Agents Chemother 2003;47:1318-1323.

-15 Woodworth JR, Nyhart EH Jr, Brier GL, Wolny JD, Black HR: Single-dose pharmacokinetics and antibacterial activity of daptomycin, a new lipopeptide antibiotic, in healthy volunteers. Antimicrob Agents Chemother 1992;36:318-325.

16 Lee BL, Sachdeva M, Chambers HF: Effect of protein binding of daptomycin on MIC and antibacterial activity. Antimicrob Agents Chemother 1991;35:2505-2508.
7 Pankuch GA, Jacobs MR, Appelbaum PC: Postantibiotic effects of daptomycin against 14 staphylococcal and pneumococcal clinical isolates. Antimicrob Agents Chemother 2003;47:3012-3014.

18 Hanberger H, Nilsson LE, Maller R, Isaksson B: Pharmacodynamics of daptomycin and vancomycin on Enterococcus faecalis and Staphylococcus aureus demonstrated by studies of initial killing and postantibiotic effect and influence of $\mathrm{Ca}^{2+}$ and albumin on these drugs. Antimicrob Agents Chemother 1991;35:1710-1716.

19 Edmiston CE Jr, Goheen MP, Seabrook GR, Johnson CP, Lewis BD, Brown KR, Towne JB: Impact of selective antimicrobial agents on staphylococcal adherence to biomedical devices. Am J Surg 2006;192:344-354.

20 Laplante KL, Mermel LA: In vitro activity of daptomycin and vancomycin lock solutions on staphylococcal biofilms in a central venous catheter model. Nephrol Dial Transplant 2007;22:2239-2246.

21 Raad II, Hanna HA, Boktour M, Chaiban G, Hachem RY, Dvorak T, Lewis R, Murray BE: Vancomycin-resistant Enterococcus faecium: catheter colonization, esp gene, and decreased susceptibility to antibiotics in biofilm. Antimicrob Agents Chemother 2005; 49:5046-5050.

22 Credito K, Lin G, Appelbaum PC: Activity of daptomycin alone and in combination with rifampin and gentamicin against Staphylococcus aureus assessed by time-kill methodology. Antimicrob Agents Chemother 2007; 51:1504-1507.

23 DeRyke CA, Sutherland C, Zhang B, Nicolau DP, Kuti JL: Serum bactericidal activities of high-dose daptomycin with and without coadministration of gentamicin against isolates of Staphylococcus aureus and Enterococcus species. Antimicrob Agents Chemother 2006;50:3529-3534.

24 Rand KH, Houck H: Daptomycin synergy with rifampicin and ampicillin against vancomycin-resistant enterococci. J Antimicrob Chemother 2004;53:530-532.

25 Rice LB, Eliopoulos GM, Moellering RC Jr: In vitro synergism between daptomycin and fosfomycin against Enterococcus faecalis isolates with high-level gentamicin resistance. Antimicrob Agents Chemother 1989;33: 470-473.

26 Rand KH, Houck HJ: Synergy of daptomycin with oxacillin and other beta-lactams against methicillin-resistant Staphylococcus aureus. Antimicrob Agents Chemother 2004;48: 2871-2875.

27 English BK, Maryniw EM, Talati AJ, Meals EA: Diminished macrophage inflammatory response to Staphylococcus aureus isolates exposed to daptomycin versus vancomycin or oxacillin. Antimicrob Agents Chemother 2006;50:2225-2237.
28 Tedesco KL, Rybak MJ: Daptomycin. Pharmacotherapy 2004;24:41-57.

29 Richter SS, Kealey DE, Murray CT, Heilmann KP, Coffman SL, Doern GV: The in vitro activity of daptomycin against Staphylococcus aureus and Enterococcus species. J Antimicrob Chemother 2003;52:123-127.

30 Johnson AP, Mushtaq S, Warner M, Livermore DM: Calcium-supplemented daptomycin Etest strips for susceptibility testing on Iso-Sensitest agar. J Antimicrob Chemother 2004;53:860-862.

31 Clinical CaLSI: Performance standards for antimicrobial susceptibility testing; 16th international supplement. CLSI Document 2006; M100-S16. Wayne, 2006.

32 Sader HS, Fritsche TR, Jones RN: Daptomycin bactericidal activity and correlation between disk and broth microdilution method results in testing of Staphylococcus aureus strains with decreased susceptibility to vancomycin. Antimicrob Agents Chemother 2006;50:2330-2336.

33 Hayden MK, Rezai K, Hayes RA, Lolans K, Quinn JP, Weinstein RA: Development of daptomycin resistance in vivo in methicillin-resistant Staphylococcus aureus. J Clin Microbiol 2005;43:5285-5287.

34 Jevitt LA, Thorne GM, Traczewski MM, Jones RN, McGowan JE Jr, Tenover FC, Brown SD: Multicenter evaluation of the Etest and disk diffusion methods for differentiating daptomycin-susceptible from nondaptomycin-susceptible Staphylococcus aureus isolates. J Clin Microbiol 2006;44: 3098-3104.

35 Clinical CaLSI: Performance standards for antimicrobial disk susceptibility tests; approved standard, ed 9. CLSI Document 2006; M2-A9. Wayne, 2006.

36 Streit JM, Jones RN, Sader HS: Daptomycin activity and spectrum: a worldwide sample of 6737 clinical Gram-positive organisms. J Antimicrob Chemother 2004;53:669-674.

37 Critchley IA, Draghi DC, Sahm DF, Thornsberry C, Jones ME, Karlowsky JA: Activity of daptomycin against susceptible and multidrug-resistant Gram-positive pathogens collected in the SECURE study (Europe) during 2000-2001. J Antimicrob Chemother 2003;51:639-649.

38 King A, Phillips I: The in vitro activity of daptomycin against 514 Gram-positive aerobic clinical isolates. J Antimicrob Chemother 2001;48:219-223.

39 Piper KE, Steckelberg JM, Patel R: In vitro activity of daptomycin against clinical isolates of Gram-positive bacteria. J Infect Chemother 2005;11:207-209.

40 Tyrrell KL, Citron DM, Warren YA, Fernandez HT, Merriam CV, Goldstein EJ: In vitro activities of daptomycin, vancomycin, and penicillin against Clostridium difficile, C. perfringens, Finegoldia magna, and Propionibacterium acnes. Antimicrob Agents Chemother 2006;50:2728-2731. 
-41 Rybak MJ, Hershberger E, Moldovan T, Grucz RG: In vitro activities of daptomycin, vancomycin, linezolid, and quinupristindalfopristin against Staphylococci and Enterococci, including vancomycin-intermediate and -resistant strains. Antimicrob Agents Chemother 2000;44:1062-1066.

-42 Goldstein EJ, Citron DM, Merriam CV, Warren YA, Tyrrell KL, Fernandez HT: In vitro activities of the new semisynthetic glycopeptide telavancin (TD-6424), vancomycin, daptomycin, linezolid, and four comparator agents against anaerobic gram-positive species and Corynebacterium spp. Antimicrob Agents Chemother 2004;48:2149-2152.

43 Dvorchik B, Damphousse D: Single-dose pharmacokinetics of daptomycin in young and geriatric volunteers. J Clin Pharmacol 2004;44:612-620.

-44 Dvorchik B: Moderate liver impairment has no influence on daptomycin pharmacokinetics. J Clin Pharmacol 2004;44:715-722.

45 Benvenuto M, Benziger DP, Yankelev S, Vigliani G: Pharmacokinetics and tolerability of daptomycin at doses up to 12 milligrams per kilogram of body weight once daily in healthy volunteers. Antimicrob Agents Chemother 2006;50:3245-3249.

-46 Dvorchik B, Arbeit RD, Chung J, Liu S, Knebel W, Kastrissios H: Population pharmacokinetics of daptomycin. Antimicrob Agents Chemother 2004;48:2799-2807.

47 Dvorchik BH, Damphousse D: The pharmacokinetics of daptomycin in moderately obese, morbidly obese, and matched nonobese subjects. J Clin Pharmacol 2005;45: 48-56.

48 Pai MP, Norenberg JP, Anderson T, Goade D, Rodvold KA, Telepak R, Mercier RC: Daptomycin pharmacokinetics in morbidly obese subjects. 46th Intersci Conf on Antimicrob Agents Chemother 2006, A-1947.

-49 Wise R, Gee T, Andrews JM, Dvorchik B, Marshall G: Pharmacokinetics and inflammatory fluid penetration of intravenous daptomycin in volunteers. Antimicrob Agents Chemother 2002;46:31-33.

50 Gerber P, Stucki A, Acosta F, Cottagnoud M, Cottagnoud P: Daptomycin is more efficacious than vancomycin against a methicillin-susceptible Staphylococcus aureus in experimental meningitis. J Antimicrob Chemother 2006;57:720-723.

-51 Cottagnoud P, Pfister M, Acosta F, Cottagnoud M, Flatz L, Kuhn F, Muller HP, Stucki A: Daptomycin is highly efficacious against penicillin-resistant and penicillin- and quinolone-resistant pneumococci in experimental meningitis. Antimicrob Agents Chemother 2004;48:3928-3933.

52 Ahmad QI, Mankowski M, Girish SR: Safety and tolerability of daptomycin iv bolus injections in healthy adult volunteers. 46th Intersci Conf on Antimicrob Agents Chemother 2006, A-1948.
53 Trotman RL, Williamson JC, Shoemaker DM, Salzer WL: Antibiotic dosing in critically ill adult patients receiving continuous renal replacement therapy. Clin Infect Dis 2005;41:1159-1166.

54 Cunha BA, Eisenstein LE, Hamid NS: Pacemaker-induced Staphylococcus aureus mitral valve acute bacterial endocarditis complicated by persistent bacteremia from a coronary stent: cure with prolonged/highdose daptomycin without toxicity. Heart Lung 2006;35:207-211.

55 Arbeit RD, Maki D, Tally FP, Campanaro E, Eisenstein BI: The safety and efficacy of daptomycin for the treatment of complicated skin and skin-structure infections. Clin Infect Dis 2004;38:1673-1681.

56 Lipsky BA, Stoutenburgh U: Daptomycin for treating infected diabetic foot ulcers: evidence from a randomized, controlled trial comparing daptomycin with vancomycin or semi-synthetic penicillins for complicated skin and skin-structure infections. J Antimicrob Chemother 2005;55:240-245.

57 Fowler VG Jr, Boucher HW, Corey GR, Abrutyn E, Karchmer AW, Rupp ME, Levine DP, Chambers HF, Tally FP, Vigliani GA, Cabell CH, Link AS, DeMeyer I, Filler SG, Zervos M, Cook P, Parsonnet J, Bernstein JM, Price CS, Forrest GN, Fatkenheuer G, Gareca M, Rehm SJ, Brodt HR, Tice A, Cosgrove SE: Daptomycin versus standard therapy for bacteremia and endocarditis caused by Staphylococcus aureus. N Engl J Med 2006; 355:653-665.

58 Segreti JA, Crank CW, Finney MS: Daptomycin for the treatment of gram-positive bacteremia and infective endocarditis: a retrospective case series of 31 patients. Pharmacotherapy 2006;26:347-352.

59 Sakoulas G, Brown JE, Lamp KC, Friedrich LV: Efficacy and safety of daptomycin in patients treated for non-catheter related bacteremia. 46th Intersci Conf on Antimicrob Agents Chemother 2006, L-1536.

60 Poutsiaka DD, Skiffington S, Miller KB, Hadley S, Snydman DR: Daptomycin in the treatment of vancomycin-resistant Enterococcus faecium bacteremia in neutropenic patients. J Infect 2006;54:567-571.

61 Shah M, Murillo JL: Successful treatment of Corynebacterium striatum endocarditis with daptomycin plus rifampin. Ann Pharmacother 2005;39:1741-1744.

62 Lamp KC, Friedrich LV: Clinical experience with daptomycin for the treatment of osteomyelitis in patients with post-therapy follow-up. 46th Intersci Conf on Antimicrob Agents Chemother 2006, L-1557.

63 Forrest G, Donovan B, Lamp K, Friedrich L: Daptomycin use in patients with septic arthritis: post-marketing experience from CORE 2005. 46th Intersci Conf on Antimicrob Agents Chemother 2006, A-1556.
64 Finney MS, Crank CW, Segreti J: Use of daptomycin to treat drug-resistant Gram-positive bone and joint infections. Curr Med Res Opin 2005;21:1923-1926.

65 Rao N, Regalla DM: Uncertain efficacy of daptomycin for prosthetic joint infections: a prospective case series. Clin Orthop Relat Res 2006;451:34-37.

66 McKinnon PS, Krueger TS, Lamp KC, Friedrich LV, Brown JE: Determinants of clinical outcomes in patients treated with daptomycin for gram-positive infections. 46th Intersci Conf on Antimicrob Agents Chemother 2006, K-782.

67 Silverman JA, Mortin LI, Vanpraagh AD, Li T, Alder J: Inhibition of daptomycin by pulmonary surfactant: in vitro modeling and clinical impact. J Infect Dis 2005;191:21492152.

68 Kephart PA, Esposito AL: Comparison of the investigational drug, LY146032, with vancomycin in experimental pneumonia due to methicillin-resistant Staphylococcus aureus. J Antimicrob Chemother 1988;21:33-39.

69 Silverman JA, Oliver N, Andrew T, Li T: Resistance studies with daptomycin. Antimicrob Agents Chemother 2001;45:1799-1802.

70 Mortin LI, Friedman L, Li T, Van Praagh ADG, Zhang S, Zhang X, Alder JD: Daptomycin efficacy vs. laboratory-derived mutant Staphylococcus aureus with MICs of 4-16 $\mu \mathrm{g} / \mathrm{mL}$. 46th Intersci Conf on Antimicrob Agents Chemother (ICAAC) 2006;51: 1787-1794.

71 Rose WE, Rybak MJ, Kaatz GW: Evaluation of daptomycin treatment of Staphylococcus aureus bacterial endocarditis: an in vitro and in vivo simulation using historical and current dosing guidelines. 46th Intersci Conf on Antimicrob Agents Chemother 2006, A630.

72 Lewis JS 2nd, Owens A, Cadena J, Sabol K, Patterson JE, Jorgensen JH: Emergence of daptomycin resistance in Enterococcus faecium during daptomycin therapy. Antimicrob Agents Chemother 2005;49:16641665.

73 Skiest DJ: Treatment failure resulting from resistance of Staphylococcus aureus to daptomycin. J Clin Microbiol 2006;44:655-656.

74 Marty FM, Yeh WW, Wennersten CB, Venkataraman L, Albano E, Alyea EP, Gold HS, Baden LR, Pillai SK: Emergence of a clinical daptomycin-resistant Staphylococcus aureus isolate during treatment of methicillin-resistant Staphylococcus aureus bacteremia and osteomyelitis. J Clin Microbiol 2006;44:595597.

75 Hirschwerk D, Ginocchio CC, Bythrow M, Condon S: Diminished susceptibility to daptomycin accompanied by clinical failure in a patient with methicillin-resistant Staphylococcus aureus bacteremia. Infect Control Hosp Epidemiol 2006;27:315-317. 
76 Sharma M, Riederer K, Khatib R: Decreasing daptomycin susceptibility during treatment of Staphylococcus aureus bacteremia. 46th Intersci Conf on Antimicrob Agents Chemother 2006, E-728.

77 Luperchio SA, Mortin LI, Silverman JA, Chambers HF, Alder JD: MIC shifts in patients treated for Staphylococcus aureus bacteremia and infective endocarditis. 46th Intersci Conf on Antimicrob Agents Chemother 2006, L-1206.

78 Rose WE, Rybak MJ, Sakoulas G: Daptomycin activity on S. aureus isolates pre and post exposure to vancomycin during treatment for bacterial endocarditis. 46th Intersci Conf on Antimicrob Agents Chemother 2006, C1-686.

79 Mariani PG, Sader HS, Jones RN: Development of decreased susceptibility to daptomycin and vancomycin in a Staphylococcus aureus strain during prolonged therapy. J Antimicrob Chemother 2006;58:481-483.

80 Cui L, Tominaga E, Neoh HM, Hiramatsu K: Correlation between reduced daptomycin susceptibility and vancomycin resistance in vancomycin-intermediate Staphylococcus aureus. Antimicrob Agents Chemother 2006;50:1079-1082.
81 Kosowska-Shick K, Julian K, Brumbach A, Curley E, Whitener C, Parent L, Bogdanovich T: Characteristics of a $S$. aureus strain causing endocarditis which acquired VISA and daptomycin-resistant phenotypes after daptomycin therapy. 46th Intersci Conf on Antimicrob Agents Chemother 2006, E-727.

82 Sheth A, Carpenter CF, Robinson-Dunn B: Reduced vancomycin susceptibility and daptomycin nonsusceptibility associated with treatment failure in 2 cases of MRSA bacteremia. 46th Intersci Conf on Antimicrob Agents Chemother 2006, C2-1159.

83 Bell JM, Walter LJ, Turnidge JD, Jones RN: Vancomycin hetero-resistance has a small but significant effect on the daptomycin minimum inhibitory concentration of Staphylococcus aureus. 46th Intersci Conf on Antimicrob Agents Chemother 2006, D-814.

84 Munoz-Price LS, Lolans K, Quinn JP: Emergence of resistance to daptomycin during treatment of vancomycin-resistant Enterococcus faecalis infection. Clin Infect Dis 2005;41:565-566.

85 Stubbings WJ, Bell R, Ryder V, Bedi M, Keen J, Chopra I: Proteomic investigation of the mode of action of daptomycin in Staphylococcus aureus. 46th Intersci Conf on Antimicrob Agents Chemothe 2006, C1-683.

86 Kosowska-Shick K, Roos M, Labischinski H, Whitener C, Julian K, Appelbaum PC: Cell wall analysis of a series of related clinical Staphylococcus aureus isolates with decreasing susceptibility towards vancomycin and daptomycin. 46th Intersci Conf on Antimicrob Agents Chemother 2006,C1-6.
7 Patel JB, Jevitt LA, Hageman J, McDonald LC, Tenover FC: An association between reduced susceptibility to daptomycin and reduced susceptibility to vancomycin in Staphylococcus aureus. Clin Infect Dis 2006;42: 1652-1653.

88 Echevarria K, Datta P, Cadena J, Lewis JS 2nd: Severe myopathy and possible hepatotoxicity related to daptomycin. J Antimicrob Chemother 2005;55:599-600.

89 Veligandla SR, Louie KR, Malesker MA, Smith PW: Muscle pain associated with daptomycin. Ann Pharmacother 2004;38:18601862.

90 Papadopoulos S, Ball AM, Liewer SE, Martin CA, Winstead PS, Murphy BS: Rhabdomyolysis during therapy with daptomycin. Clin Infect Dis 2006;42:e108-e110.

91 Kazory A, Dibadj K, Weiner ID: Rhabdomyolysis and acute renal failure in a patient treated with daptomycin. J Antimicrob Chemother 2006;57:578-579.

92 Bhavnani SM, Ambrose PG, Oleson FB, Drusano GL: Toxicodynamics of daptomycin in patients with bacteremia and/or endocarditis. 46th Intersci Conf on Antimicrob Agents Chemother 2006, A-655.

93 Farmer JA, Torre-Amione G: Comparative tolerability of the HMG-CoA reductase inhibitors. Drug Saf 2000;23:197-213.

94 Hayes D Jr, Anstead MI, Kuhn RJ: Eosinophilic pneumonia induced by daptomycin. J Infect 2007;54:e211-e213. 